\title{
CHINESE REMAINDER THEOREMS AND GALOIS MODULES
}

\author{
NEIL ORMEROD \\ (Received 17 August, 1984; revised 13 November 1984) \\ Communicated by G. Brown
}

\begin{abstract}
This paper studies the relationship between a normal algebraic extension $L$ of an algebraic number field $K$, viewed as a Galois module, and valuations of the field $L$. In particular, the paper seeks to establish a relationship between Galois submodules of $L$ and certain types of Chinese Remainder Theorems.
\end{abstract}

1980 Mathematics subject classification (Amer. Math. Soc.): 12 A 55.

Key words and phrases: Galois modules, Chinese Remainder Theorems.

\section{Introduction}

The purpose of this paper is to consider the possibility of introducing arithmetically defined norms on naturally arising Galois modules. Having introduced such norms, one can ask how independent inequivalent norms are; that is, there arises the question of Chinese Remainder Theorems. As we shall see, the possibility of satisfying some type of Chinese Remainder Theorem is linked to the representation type of the module considered. The bulk of this paper is concerned with basic machinery and results. The final section, however, deals with possible applications of the type of results we are considering.

As a model of much of what follows, consider the following situation. Let $L$ be a finite normal extension of $K$, a finite algebraic extension of $\mathbb{Q}$, and let $G=G(L / K)$. Then it is well known that $L$ has a normal basis over $K$, so that, as a $K G$ module, $L$ is the left regular representation. Let us prove this using the Chinese Remaider Theorem for $L$.

(c) 1986 Australian Mathematical Society $0263-6115 / 86 \$ A 2.00+0.00$ 
THEOREM 1. Let $L$ be a finite normal extension of $K$, with $K$ a finite algebraic extension of $\mathbb{Q}$, and let $G=G(L / K)$. Then as a $K G$ module $L$ is the left regular representation, i.e. $L$ has a normal basis.

Proof. By the Chinese Remainder Theorem for $L$ we have

$$
K_{v} \otimes_{K} L \cong \bigoplus_{w \mid v} L_{w}
$$

(cf. [2, pages $54 \mathrm{ff}]$ ). Since $G$ acts transitively on the right-hand side, we see that the right-hand side forms a system of imprimitivity for the module $K_{v} \otimes_{K} L$. Hence the left-hand side is an induced $G_{w}$ module, where $G_{w}$ is the decomposition group of $L_{w}$. However, by Cebotarev's Theorem [7, page 165], there exist primes $v$ such that, for $w \mid v$, we have $G_{w}=1$ and $L_{w}=K_{v}$. But then $K_{v} \otimes_{K} L$ is simply the left regular representation of $G$, and hence so is $L$.

Although we have had to invoke some rather powerful results to obtain a normal basis theorem, it does point out the rather strong connection between the arithmetic of a field, via the Chinese Remainder Theorem, and its module structure.

\section{Norms on Galois modules}

Let $L$ be a finite normal extension of a field $K$, where $K$ is some finite algebraic extension of $\mathbb{Q}$. Let $M$ be some $K$ subspace of $L$. For $w$, any finite prime of $L$, define a norm on $M$ as follows. For $x \in M$, let

$$
\|x\|_{w}=|x|_{w}^{n_{v}^{-1}}, \quad n_{v}=\left[L_{w}: K_{v}\right]
$$

It is clear that this defines a norm on $M$, i.e. that

1. $\|x\|_{w}>0 \forall x \neq 0$,

2. $\|a x\|_{w}=|a|_{v}\|x\|_{w} \forall a \in K, w \mid v$, and

3. $\|x+y\|_{w} \leqslant\|x\|_{w}+\|y\|_{w}, x, y \in M$.

Of course different $w$ may induce equivalent norms on $M$, and we write $w_{1}-w_{2}$, so that we obtain an equivalence class $W_{1}=\left\{w \mid w\right.$ a prime of $\left.L, w \sim w_{1}\right\}$.

The following lemma clarifies the obvious observation that if $w_{1} \mid v_{1}$ and $w_{2} \mid v_{2}$, for $v_{1}, v_{2}$ distinct primes in $K$, then $w_{1}+w_{2}$.

LEMMA 1. Let \|\|$_{w_{1}}, \ldots,\|\|_{w_{r}}$ be a set of norms, with $w_{i}$ lying over distinct primes $v_{i}$ of $K$. Then for all $a_{1}, \ldots, a_{r} \in M$ and $\varepsilon>0$, there exists $z \in M$ such that

$$
\left\|z-a_{i}\right\|_{w}<\varepsilon
$$

for all $w \mid v_{i}$. 
Proof. By the Chinese Remainder Theorem for $K$, there exist pairs of algebraic integers in $\mathcal{O}_{K}$, such that, given any $\varepsilon^{\prime}>0$, we have

$$
X_{1 i}+X_{2 i}=1 \text {, }
$$

where $\left|X_{1 i}\right|_{v_{i}}<\varepsilon^{\prime},\left|X_{2 i}\right|_{v_{j}}<\varepsilon^{\prime}$ for all $j \neq i$ (cf. [2, page 48]). Put $z=\sum_{j=1}^{r} X_{2 j} a_{j}$. Then

$$
\begin{aligned}
\left\|z-a_{i}\right\|_{w} & =\left\|\left(X_{2 i}-1\right) a_{i}+\sum_{j \neq i} X_{2 j} a_{j}\right\|_{w} \\
& =\left\|X_{1 i} a_{i}+\sum_{j \neq i} X_{2 j} a_{j}\right\|_{w} \\
& \leqslant 2^{r} \max _{j \neq i}\left\{\left\|X_{1 i} a_{i}\right\|_{w},\left\|X_{2 j} a_{j}\right\|_{w}\right\} \\
& \leqslant \varepsilon^{\prime} 2^{r} \max \left\{\left\|a_{j}\right\|_{w}\right\},
\end{aligned}
$$

for all $w \mid v$. However, we can make the right-hand side as small as we please.

Let us further suppose that $M$ is $G$-invariant, where $G=G(L / K)$. Thus $M$ becomes a $K G$ module. For a fixed prime $v$ of $K, G$ acts transitively on the primes $w \mid v$ of $L$, so that $G$ also acts transitively on the equivalence classes $W$ of norms on $M$. Put $\Gamma_{W}=\{g \in G \mid g W=W\}$. Then $\Gamma_{W}$ plays the role of the usual decomposition subgroup $G_{w}=\{g \in G \mid g w=w\}$. The following observations are trivial.

01. $G_{w}$ is a subgroup of $\Gamma_{W}$ if $w \in W$.

02. $\left|\Gamma_{W}: G_{w}\right|$ is the number of $w$ in a given equivalence class.

03. $\left|G: \Gamma_{W}\right|$ is the number of inequivalent norms on $M$ lying over $v$.

04. $\Gamma_{g W}=g \Gamma_{W} g^{-1}$.

Our major aim will be to show how the arithmetic properties of our norms, as given in terms of Chinese Remainder-type Theorems, are related to the representation type of the $G K$ module $M$.

Notationally I shall pass from equivalence classes $W$ to elements $w \in W$ and vice-versa, whichever seems more natural in a given setting.

\section{Chinese Remainder Theorems}

I shall say that $M$ satisfies the Chinese Remainder Theorem (CRT) if the following halds:

Let \|\|$_{w_{1}}, \ldots,\|\|_{w_{r}}$ be inequivalent norms on $M$. For all $a_{1}, \ldots, a_{r}$ in $M$, and for all $\varepsilon>0$, there exists $z \in M$ such that $\left\|z-a_{i}\right\|_{w_{i}}<\varepsilon$, i.e. the image of $M$ under the diagonal mapping of $M \rightarrow \oplus_{i} M_{w_{i}}$ defined by $z \rightarrow(z, \ldots, z)$ is dense, where $M_{w}$ is the completion of $M$ under the norm \|\|$_{w}$. 
As in Theorem 1, we have the following.

THEOREM 2. If $M$ is a G-invariant subspace of $L$ and satisfies the CRT, then $M$ is monomial.

Proof. Since $M$ satisfies the CRT we have, as in Theorem 1, that

$$
K_{v} \otimes_{K} M \equiv \bigotimes_{w \mid v} M_{w},
$$

where the sum is over all inequivalent norms induced by $w \mid v$. Thus $K_{v} \otimes_{K} M$ is an induced $K_{v} \Gamma_{W}$ module. Again by Cebotarev's Theorem there exist primes $v$ such that $K_{v}=L_{w}$. However, we clearly have an injection $M_{w} \rightarrow L_{w}$, so that $\operatorname{dim} M_{w}=1$. Thus $K_{v} \otimes_{K} M$, and hence $M$, is monomial.

Thus the CRT is a fairly restrictive condition. Indeed, the following lemma from group theory indicates some further restrictions.

LEMMA 2. Let $H$ be a normal subgroup of $G$, and let $\theta$ be a character of $H$. Assume that $\theta^{G}$ is irreducible. Then $\left.\theta^{G}\right|_{H}$ is multiplicity free.

Proof. By a standard corollary to Mackay's irreducibility criterion, $\theta^{G}$ is irreducible if and only if $\theta$ and $\theta^{x}$ are distinct for all $x \in H$, and $\theta$ is irreducible, where $\theta^{x}(g)=\theta\left(x^{-1} g x\right)$ (cf. [6, Proposition 23 and the following corollary, pages 59-60]).

However, we clearly have by the definition of induced representations that $\left.\theta^{G}\right|_{H}=\sum_{x \in G / H} \theta^{x}$. The lemma then follows immediately.

COROLlaRY 1. Suppose $K$ is a splitting field for the representations of $\Gamma_{W}$, a normal subgroup of $G$, where if $w \in W$, then $L_{w}=K_{v}$. Then $M$ is irreducible and satisfies the CRT if and only if $M$ is one dimensional.

Proof. First, it is clear that if $M$ is one dimensional, then it is irreducible and satisfies the CRT. Conversely, by Theorem 2 we have that $M$ is an induced $\Gamma_{W}$ module and that $K_{v} \otimes_{K} M \cong \oplus_{w \mid v} M_{w}$. Fix $v$ such that, if $w \mid v$, then $K_{v}=L_{w}$. Then we also have a decomposition $M=\oplus M_{i}$ into irreducible $K \Gamma_{W_{1}}$ modules, by our assumption on $K$, and this decomposition is unique because of the multiplicity free result in Lemma 2 . Thus

$$
K_{v} \otimes_{K} M \cong \bigoplus K_{v} \otimes_{K} M_{i} \cong \bigoplus_{w \mid v} M_{w} .
$$

Now consider the projection onto the first component of the extreme right-hand side $\oplus K_{v} \otimes_{K} M_{i} \rightarrow M_{w_{1}}$. This is a homomorphism of $K_{v} \Gamma_{W_{1}}$ modules, and hence the kernel is a $K_{v} \Gamma_{W_{1}}$ module. By the uniqueness of the left-hand side, some 
$K_{v} \otimes_{K} M_{i}$ must be annihilated. But this is impossible since we have natural injections $M_{i} \rightarrow M \rightarrow M_{w}$. Thus we must have $K_{v} \otimes_{K} M \cong M_{w_{1}}$ and by our choice of $v$, the right-hand side is clearly one-dimensional.

Note that some such condition on $K$ as given in the corollary is necessary. For instance, the first example in Section 5 clearly satisfies the CRT and is irreducible. However, since $\mathbf{Q}$ does not contain the cube roots of unity, $K_{2}$ cannot be split into absolutely irreducible submodules over $\mathbb{Q}$ when $\Gamma_{w} \cong\langle(123)\rangle$.

Let us seek a more general form of Chinese Remainder Theorem. Let $W$ denote an equivalence class of norms on $M$. Clearly then $g W$ is another equivalence class. For $H$ a subgroup of $G$, let $H W$ denote the $H$ orbit of $W$. Then $g_{1} W$ and $g_{2} W$ are both in $H W$ if and only if $g_{1}$ and $g_{2}$ lie in the same $\left(H, \Gamma_{W}\right)$ double coset. Then $M$ is said to satisfy the $H$-Chinese Remainder Theorem $(H-C R T)$ if the following holds:

Let \|\|$_{w_{1}}, \ldots,\|\|_{w_{r}}$ be norms which belong to distinct $H$ orbits $H W_{i}$. For all $a_{1}, \ldots, a_{r} \in M$, and for all $\varepsilon>0$, there exists $z \in M$ such that $\left\|z-a_{i}\right\|_{w}<\varepsilon$ for all $w \in H W_{i}$.

The motivation for considering such a definition is that the primes of the intermediate field $F$, the fixed field of $H$, are naturally indexed by double cosets $\left(H, G_{w}\right)$. Notice that if $M$ satisfies the $H$-CRT, then it also satisfies the $H^{g}$-CRT for all $g \in G$. First consider the situation when $H$ is a normal subgroup of $G$.

THEOREM 3. Let $H$ be a normal subgroup of $G$, and suppose that $M$ satisfies the $H-C R T$. Then $M$ is an induced $H \Gamma_{W}$ module.

Proof. First note that if $H$ is normal, then the double sets $\left(H, \Gamma_{W}\right)$ correspond to (single) cosets of the subgroup $H \Gamma_{W}$. Now define a new norm on $M$ by

$$
\|x\|_{H W}=\max _{w \in H W}\|x\|_{w} .
$$

Let $M_{H W}$ denote the completion of $M$ in the resulting topology. Then, again by the $H$-CRT, we have that $K_{v} \otimes_{K} M \cong \oplus_{i} M_{H W_{i}}$, where the sum runs over all $H$-orbits of equivalence classes lying over $v$. Again the right-hand side forms a system of imprimitivity with decomposition group $H \Gamma_{W}$, so that $M$ is an induced $H \Gamma_{W}$ module.

Again, as with Theorem 2, the following corollary indicates some of the limits of the theorem.

COROLlaRY 2. Suppose $K$ is a splitting field for the representations of the subgroup $H \Gamma_{W}$ normal in $G$. Then $M$ is irreducible and satisfies the H-CRT only if there is one $H$-orbit of inequivalent norms lying over the prime $v$ of $K$. 
Proof. This follows as in Corollary 1.

Now let us turn our attention to the case when $H$ is not normal in $G$. The above proof breaks down because the relationship $K_{v} \otimes_{K} M \cong \oplus_{i} M_{H W_{i}}$ no longer gives rise to a system of imprimitivity since $g: M_{H W} \rightarrow M_{H^{8} g}$. All we can say is that the right hand terms are $H$-invariant. If, however, we further assume that $\Gamma_{W}=1$, then we have that $H W \cap H^{g} g W=\varnothing$ unless $g \in H$. With these observations we may prove the following.

THEOREM 4. Assume that for some $w$, we have $\Gamma_{W}=1$. If $M$ satisfies the $H$-CRT, then $M$ is an induced $H$ module.

Proposition. As before we have that $K_{v} \otimes_{K} M \cong \oplus_{i} M_{H W_{i}}$, as well as

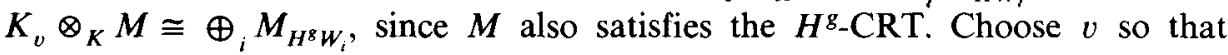
$\Gamma_{W_{1}}=1$. Now let $G=\bigcup g_{i} H$ be a coset decomposition of $G$, and consider the mapping

$$
K_{v} \otimes_{K} M \rightarrow \underset{g \in G / H}{\bigoplus_{H}} M_{H^{g} W_{1}}
$$

obtained by summing over the projections $K_{v} \otimes_{K} M \rightarrow M_{H^{g} g W_{1}}$. Since $\bigcup_{g \in G / H} H^{g} g W_{1}$ exhausts all divisors of $v$, the mapping is injective. Moreover, since we can solve for $x \in K_{v} \otimes_{K} M$, where

$$
x \mapsto(0, \ldots, 0, y, 0, \ldots, 0) \in \underset{g \in G / H}{\bigoplus} M_{H^{g} g W_{1}},
$$

by the corresponding solution

$$
x \mapsto(0, \ldots, 0, y, 0, \ldots, 0) \in \bigoplus_{i} M_{H^{s} W_{i}},
$$

where the non-zero term occurs in the $W_{i}=g W_{1}$ component, we see that the mapping is surjective. Thus we have an isomorphism

$$
K_{v} \otimes_{K} M \cong \bigoplus_{g \in G / H} M_{H^{g} g W_{1}},
$$

where, now the right-hand side forms a system of imprimitivity with decomposition group $H$. Thus $M$ is an induced $H$ module.

\section{Converse results}

Of course what has been shown in the previous section is of little interest if no modules can be shown to satisfy any type of Chinese Remainder Theorem. Our aim now will be to construct modules which do just that. 
Let $H$ be a subgroup of $G$, with fixed field $F$. For $M^{\prime}$ an $F H$ module and any $g \in G, g M^{\prime}$ is an $F^{g} H^{g}$ module. Now put $M=\Sigma_{g \in G / H} g M^{\prime}$. Then $M$ is clearly a $K G$ module with dimension less than or equal to $[G: H]^{2} \operatorname{dim} M^{\prime}$.

THEOREM 5. The module $M$ constructed above satisfies the $H$-CRT for $H$ normal in $G$.

Proof. Let $x$ be in $M$, say. Then $x=\sum_{g_{i} \in G / H} g_{i} x_{i}$ for some $x_{i} \in M^{\prime}$, so that

$$
\begin{aligned}
\|x\|_{w} & =\left\|\sum g_{i} x_{i}\right\|_{w} \\
& \leqslant 2^{m} \max _{i}\left\|g_{i} x_{i}\right\|_{w} \quad(\text { where } m=[G: H]) \\
& =2^{m} \max _{i}\left\|x_{i}\right\|_{g_{i}-{ }^{-1}} .
\end{aligned}
$$

Thus, given $a_{1}, \ldots, a_{r} \in M$ with $a_{i}=\sum_{j} g_{j} a_{i j}$, we have that

$$
\left\|x-a_{i}\right\|_{w_{i}} \leqslant 2^{m} \max _{j}\left\|x_{j}-a_{j j}\right\|_{g_{j}^{-1} w_{i}} .
$$

Thus we need only consider approximations of the type $\left\|x_{j}-a_{i j}\right\|_{g_{j}^{-1} w_{i}}$. However, by Lemma 1, if $\left\{g_{j}^{-1} w_{i}\right\}_{i=1}^{r}$ lie over distinct primes $v^{\prime}$ of $F$, solutions $x_{j}$ in $M^{\prime}$ can be found such that

$$
\left\|x_{j}-a_{i j}\right\|_{g_{j}^{-1} w}<\varepsilon
$$

for all $g_{j}^{-1} w \mid v^{\prime}$, if we are given that $g_{j}^{-1} w_{i} \mid v^{\prime}$. Now if $w_{1}, \ldots, w_{r}$ lie in distinct $H$ orbits, so also do $g_{j}^{-1} w_{1}, \ldots, g_{j}^{-1} w_{r}$, since $H$ is normal, so they lie over distinct primes of $v^{\prime}$ of $F$. Thus solutions $x_{j}$ to (*) exist such that

$$
\left\|x-a_{i}\right\|_{w} \leqslant 2^{m} \max _{j}\left\|x_{j}-a_{i j}\right\|_{g_{j}^{-1} w} \leqslant 2^{m} \varepsilon
$$

for all $w$ in the same $H$-orbit as $w_{i}$.

It is not at all clear how to generalise this to the case where $H$ is not normal. As indicated by the nature of Theorem 4, when $H$ is not normal extra conditions must be applied, and so we would expect the situation to be more complex. Perhaps a different construction is required.

\section{Examples}

Perhaps it would be wise to conclude with some concrete examples, which hopefully will illustrate some of what is and what is not possible. 
EXAMPle 1. Let $L=\mathbf{Q}(\omega, \sqrt[3]{2})$, where $\omega^{3}=1, \omega \neq 1$, and let $K=\mathbf{Q}, F=$ $\mathbf{Q}(\omega)$, and $M=\sqrt[3]{2} \mathbf{Q}(\omega)$. For simplicity let us consider valuations rather than norms. Let $w$ be a prime of $L$. For $x \in M$, we have $x=\sqrt[3]{2} z$, where $z \in \mathbf{Q}(\omega)$, so that $|x|_{w}=|\sqrt[3]{2}|_{w}|z|_{w}$. Thus all that is important is the restriction of $w$ to $F$, so that $w_{1}$ and $w_{2}$ induce equivalent norms if and only if they lie above the same prime $v^{\prime}$ of $F$. Moreover, $M$ can be obtained from the type of construction given in Section 4, so that $M$ satisfies the $H$-CRT, where $H=G(L / F)$. Moreover, for non-ramified primes, $\Gamma_{W}=H$ if there are two conjugate primes $v_{1}^{\prime}$ and $v_{2}^{\prime}$ of $F$ which lie over the rational prime $p$ with $w \mid p$, i.e. when $p \equiv 1 \bmod 3$. Otherwise $\Gamma_{W}=G$. Thus if $p \equiv 1 \bmod 3$ we have, for inequivalent $W_{1}$ and $W_{2}$, that $\mathbf{Q}_{P} \otimes_{\mathbf{Q}} M \cong M_{W_{1}} \oplus M_{W_{2}}$. Otherwise, for unramified $p$, we have $\mathbf{Q}_{P} \otimes_{\mathbf{Q}} M \cong$ $M_{W}$.

EXAMPLE 2. Let $L=\mathbf{Q}\left(\omega, \sqrt[3]{2}, \sqrt{\alpha_{1}}, \sqrt{\alpha_{2}}\right)$, and let $K=\mathbf{Q}$, where $\alpha_{1}=$ $(1+\sqrt[3]{2})(1+\omega \sqrt[3]{2})$ and $\alpha_{2}=(1+\sqrt[3]{2})(1+\omega \sqrt[3]{2})$. Then $G(L / K) \cong S_{4}$. For convenience put $\alpha_{3}=(1+\omega \sqrt[3]{2})\left(1+\omega^{2} \sqrt[3]{2}\right)$, so that $\sqrt{\alpha_{1} \alpha_{2}}=(1+\sqrt[3]{2}) \sqrt{\alpha_{3}}$, i.e. $\sqrt{\alpha_{3}} \in L$. Consider the $\mathbf{Q} G$ module $M=\left\{x_{1} \sqrt{\alpha_{1}}+x_{2} \sqrt{\alpha_{2}}+x_{3} \sqrt{\alpha_{3}} \mid x_{i} \in \mathbf{Q}\right\}$. Then the character of $M$ is irreducible and induced from a one-dimensional representation of one of the Sylow-2 subgroups $G^{(2)}$ of $S_{4}$. I shall show that $M$ does not satisfy any $H-C R T$ for $H=1, V_{4}$, or $G^{(2)}$, where $V_{4} \cong C_{2} \times C_{2}$ is normal in $S_{4}$. If $M$ satisfies the $H$-CRT for $H=1$ or $V_{4}$, this would mean that $M$ is an induced $H \Gamma_{W}$ module. This can only be true if $H \Gamma_{W}=G^{(2)}$ or $G$. However, if $H \Gamma_{W}=G^{(2)}$ or $G$, then $M$ splits into submodules, whose characters, when restricted to $H \Gamma_{W}$, are irreducible, and the resulting decomposition is multiplicity free. Thus the conditions of Corollaries 1 and 2 are sufficiently fulfilled to say that there can only be one $H$ orbit lying over any rational prime $p$. Alternatively, if $H=G^{(2)}$, an isomorphism of the type $\mathbf{Q}_{p} \otimes_{\mathbf{Q}} M \cong \oplus_{i} M_{H W_{i}}$ is a decomposition into $H$ invariant subspaces, so once again there can only be one $H$ orbit over any prime $p$. Let us now calculate a particular $\Gamma_{W}$ and show that this is not the case.

For an unramified prime $p$, the decomposition groups $G_{w}$, where $w \mid p$, are determined by the conjugacy classes of $S_{4}$; and by Cebotarev's theorem every conjugacy class occurs. Certainly there exists a prime $w$ in $L$ such that $G_{w}=\langle\sigma\rangle$, where $\sigma\left(x_{1} \sqrt{\alpha_{1}}+x_{2} \sqrt{\alpha_{2}}+x_{3} \sqrt{\alpha_{3}}\right)=-x_{1} \sqrt{\alpha_{1}}-x_{2} \sqrt{\alpha_{2}}+x_{3} \sqrt{\alpha_{3}}$. In fact $\sigma \in V_{4}$. Then, since $G_{w}<\Gamma_{w}$, we have

$$
\begin{aligned}
\mid x_{1} \sqrt{\alpha_{1}} & +x_{2} \sqrt{\alpha_{2}}+x_{3} \sqrt{\alpha_{3}} \pm\left.\sigma\left(x_{1} \sqrt{\alpha_{1}}+x_{2} \sqrt{\alpha_{2}}+x_{3} \sqrt{\alpha_{3}}\right)\right|_{w} \\
& \leqslant \max \left\{\left|x_{1} \sqrt{\alpha_{1}}+x_{2} \sqrt{\alpha_{2}}+x_{3} \sqrt{\alpha_{3}}\right|_{w},\left|\sigma\left(x_{1} \sqrt{\alpha_{1}}+x_{2} \sqrt{\alpha_{2}}+x_{3} \sqrt{\alpha_{3}}\right)\right|_{w}\right\} \\
& =\left|x_{1} \sqrt{\alpha_{1}}+x_{2} \sqrt{\alpha_{2}}+x_{3} \sqrt{\alpha_{3}}\right|_{w} \leqslant \max \left\{\left|x_{1} \sqrt{\alpha_{1}}+x_{2} \sqrt{\alpha_{2}}\right|_{w},\left|x_{3} \sqrt{\alpha_{3}}\right|_{w}\right\} .
\end{aligned}
$$


But the first term is just $\left|2\left(x_{1} \sqrt{\alpha_{1}}+x_{2} \sqrt{\alpha_{2}}\right)\right|_{w}$ or $\left|2 x_{3} \sqrt{\alpha_{3}}\right|_{w}$, and $|2|_{w}=1$ since 2 is ramified and $w$ is unramified. Thus

$$
\begin{aligned}
\max \left\{\left|x_{1} \sqrt{\alpha_{1}}+x_{2} \sqrt{\alpha_{2}}\right|,\left|x_{3} \sqrt{\alpha_{3}}\right|\right\} & \leqslant\left|x_{1} \sqrt{\alpha_{1}}+x_{2} \sqrt{\alpha_{2}}+x_{3} \sqrt{\alpha_{3}}\right| \\
& \leqslant \max \left\{\left|x_{1} \sqrt{\alpha_{1}}+x_{2} \sqrt{\alpha_{2}}\right|,\left|x_{3} \sqrt{\alpha_{3}}\right|\right\},
\end{aligned}
$$

i.e.

$$
\left|x_{1} \sqrt{\alpha_{1}}+x_{2} \sqrt{\alpha_{2}}+x_{3} \sqrt{\alpha_{3}}\right|_{w}=\max \left\{\left|x_{1} \sqrt{\alpha_{1}}+x_{2} \sqrt{\alpha_{2}}\right|_{w},\left|x_{3} \sqrt{\alpha_{3}}\right|_{w}\right\} .
$$

Thus, for $g \in \Gamma_{W}, g$ must preserve the right-hand side.

This can only occur if $g \in G_{w}$. Thus $\Gamma_{W}=G_{w}$. This is incompatible with any of our previous possibilities, and so $M$ cannot satisfy a $H$-CRT.

It seems in general that it is too much to expect an irreducible module to satisfy some type of Chinese Remainder Theorem. This is not too disappointing when one remembers that irreducible modules are not uniquely given, whereas larger modules, the homogeneous components of a particular irreducible, are. The nicest possible conjecture would then be that if such a module were induced from an $H$ module, then it would satisfy the $H$-CRT and, moreover, that the two double coset decompositions $\cup \mathrm{Hg}_{i} \mathrm{G}_{w}$ and $\cup \mathrm{Hg}_{i} \Gamma_{W}$ are identical. This would ensure the automatic fulfillment in Theorem 4 , and it would imply that the various decompositions involved are properly indexed by primes from intermediate fields.

\section{Applications}

I shall consider two types of applications.

(a) Let $M$ be a $G$-invariant $K$-subspace of $L$. Let $\mathcal{O}_{M}=M \cap \mathcal{O}_{L}$, and let $M^{*}$ be the dual of $M$ under the bilinear mapping given by the trace map ( $M^{*}$ is the contragradient of $M)$. Define the discriminant of $M$ to be $\mathscr{D}(M) \equiv\left[D \mathcal{O}_{M}: \mathcal{O}_{M^{*}}\right]$, where $D \mathcal{O}_{M}$ is the dual of $\mathcal{O}_{M}$ (cf. [4, page $\left.11 \mathrm{ff}\right]$ ). $\mathscr{D}(M)$ is defined by localisation, i.e.

$$
\begin{aligned}
\mathscr{D}(M) \mathcal{O}_{K_{v}} & =\left[\mathcal{O}_{K_{v}} \otimes_{\mathcal{O}_{K}} D \mathcal{O}_{M}: \mathcal{O}_{K_{v}} \otimes_{\mathcal{O}_{K}} \mathcal{O}_{M^{*}}\right] \\
& =\left[D\left(\mathcal{O}_{K_{v}} \otimes_{\mathcal{O}_{K}} \mathcal{O}_{M}\right): \mathcal{O}_{K_{v}} \otimes_{\mathcal{O}_{K}} \mathcal{O}_{M^{*}}\right] .
\end{aligned}
$$

Now if $M$ and $M^{*}$ satisfy the $H$-CRT, then

$$
\begin{aligned}
& \mathcal{O}_{K_{v}} \otimes_{\mathcal{O}_{K}} \mathcal{O}_{M} \cong \bigoplus_{i} \mathcal{O}_{M_{H w_{i}}} \text {, and } \\
& \mathcal{O}_{K_{v}} \otimes_{\mathcal{O}_{K}} \mathcal{O}_{M^{*}} \cong \bigoplus_{i} \mathcal{O}_{M_{H w_{i}}^{*}},
\end{aligned}
$$


where $M_{H W}^{*}$ is the dual of $M_{H W}$, so that the local $v$ component of $\mathscr{D}(M)$ has a factorisation into certain "semi-local" components

$$
\left[D \mathcal{O}_{M_{H W}}: \mathcal{O}_{M_{H W}^{*}}\right] \text {. }
$$

If, further, the two double coset decompositions $\cup \mathrm{Hg}_{i} G_{w}$ and $\cup \mathrm{Hg}_{i} \Gamma_{W}$ are the same, then these "semi-local" components are indexed by primes in the fixed field of $H$ lying over $v$, e.g. if $M=L$ then $\mathscr{D}(M)$ is the discriminant of the extension of $L$ over $K$, and this factorisation is well known.

(b) Following Fröhlich [5], note that the Galois modules we have considered have rank 1 over $K G$. Thus $M=\nu K G$ for some $\nu \in K G$. However, if $M$ satisfies a $H$-CRT, then $K_{v} \otimes_{K} M \cong \oplus M_{H W_{i}}$. Now in terms of the $K_{v} G$ module structure of the left-hand side, we see that

$$
M_{H W_{1}}=v_{H W_{i}} K_{v}\left[H g_{i} \Gamma_{W_{1}}\right]
$$

for some fixed $\Gamma_{W_{1}}$, where $\nu_{H W_{i}} \in K_{v}\left[H_{W_{i}}\right]$, and where $H_{W_{i}}$ is the decomposition group of the double coset $\mathrm{Hg}_{i} \Gamma_{W_{i}}$. Thus

$$
K_{v} \otimes_{K} M \cong \nu K_{v} G \cong \oplus \nu_{H W_{i}} K_{v}\left[H g_{i} \Gamma_{W_{1}}\right] .
$$

Therefore, considering $\nu$ as a linear map operating on $K_{v} G$, we see that $\nu=$ $\oplus \nu_{H W_{i}}$. Moreover, if we consider $\mathcal{O}_{M}=M \cap \mathcal{O}_{L}$ as an $\mathcal{O}_{K}[G]$ module, we have again, following Frölich, that

$$
\mathcal{O}_{K_{v}} \otimes_{\mathcal{O}_{K}} \mathcal{O}_{M}=\nu \beta_{v} \mathcal{O}_{K_{v}}[G],
$$

where $\beta_{v}$ is an invertible element of $K_{v} G$. Again, a $H$-CRT for $M$ would imply that, as linear maps,

$$
\nu \beta_{v}=\oplus \nu_{H W_{i}} \beta_{H W_{i}},
$$

where

$$
\mathcal{O}_{M_{H w_{i}}} \cong \nu_{H W_{i}} \beta_{H W_{i}} \mathcal{O}_{K_{i}}\left[H g_{i} \Gamma_{W_{i}}\right],
$$

and where $\beta_{H W_{i}}$ is an invertible element of $K_{v}\left[H_{W_{i}}\right]$.

For this last process we require that the modules involved satisfy some type of projectivity condition.

\section{References}

[1] J. W. S. Cassels and A. Frohlich, Algebraic number theory (Academic Press, London, 1967).

[2] J. W. S. Cassels, "Global number fields", in [1], 42-84.

[3] A. Frohlich, Algebraic number fields (Academic Press, London, 1977). 
[4] A. Frohlich, "Local fields", in [1], 1-41.

[5] A. Frohlich, "Galois module structure", in [4], 133-192.

[6] J.-P. Serre, Linear representations of finite groups (GTM Vol. 42, Springer-Verlag, New York, 1977).

[7] J. Tate, "Global class field theory", in [1], 163-203.

Department of Mathematics

University of New South Wales

P. O. Box 1

Kensington, NSW, 2033

Australia 